\title{
REVIEW
}

\section{Improving and Expanding Research on Burnout and Stress in The Academy: An Integrative Review}

\author{
Adam N. Pate, PharmD, ${ }^{\text {a,b }}$ Brent N. Reed, PharmD, MS, ${ }^{\mathrm{c}}$ Jeff Cain, EdD, MS, ${ }^{\mathrm{d}}$ Lauren Schlesselman, MA Ed \\ Psych, PharmD ${ }^{\mathrm{e}}$ \\ ${ }^{a}$ The University of Mississippi, School of Pharmacy, University, Mississippi \\ ${ }^{\mathrm{b}}$ Editorial Board Member, American Journal of Pharmaceutical Education, Arlington, Virginia \\ ${ }^{\mathrm{c}}$ University of Maryland, School of Pharmacy, Baltimore, Maryland \\ ${ }^{\mathrm{d}}$ University of Kentucky, College of Pharmacy, Lexington, Kentucky \\ ${ }^{\mathrm{e}}$ University of Connecticut, Center for Excellence in Teaching and Learning, Storrs, Connecticut
}

Corresponding Author: Adam N. Pate, University of Mississippi, School of Pharmacy, PO Box 1848, University, MS 38677. Tel: 662-915-8747. Email: anpate@olemiss.edu

Submitted October 4, 2021; accepted January 21, 2022; ePublished January 2022

Objective. To conduct an integrative review of existing literature evaluating burnout and stress to identify reliable, valid, psychometrically sound survey instruments that are frequently utilized in published studies and to provide best practices in conducting burnout and stress research within academic pharmacy.

Findings. 491 articles were reviewed finding 11 validated reliable surveys most frequently cited in literature that can be used in future burnout and stress research. Frequent misunderstandings and misuse of burnout and stress terminology along with inappropriate measurement were noted as well. Additionally, a variety of useful websites were identified during the review. Lastly, a relative dearth of published research evaluating organizational solutions to burnout and stress beyond personal factors, ie, resilience was identified.

Summary. Burnout and stress among student pharmacists, faculty, and staff is an important research area that necessitates more robust, rigorous evaluation using validated reliable surveys with appropriate contextualization within psychological frameworks and theory. Future research evaluating organizational-level attempts to remedy sources of burnout and stress is also needed.

Keywords: burnout, stress, well-being

\section{INTRODUCTION}

In recent years, greater attention has been focused on personal well-being including substantial effort devoted to evaluating stress and burnout of student pharmacists, faculty, and staff. Studies have attempted to quantify and compare the stress and burnout experienced by students based on year in the program, gender, ethnicity, or in comparison to other populations. ${ }^{1-5}$ In addition to quantifying and identifying stress and burnout, research has also explored both personal and organizational impact of stress and burnout on personal wellbeing and organizational effectiveness with compelling findings regarding the breadth of impact that burnout and stress can have. ${ }^{6-10}$ Unfortunately, there is a relative dearth of research especially in pharmacy education regarding interventions with resulting impact on faculty, staff, and student overall well-being,etc.

Noticing stress or burnout among students or colleagues is often the beginning of these research projects, but thoughtful consideration is required to research these observations and meaningfully contribute to and expand upon existing literature. Frequently, scholars who begin research in this area quickly identify the challenges and complexity inherent to this research. Unfortunately, many of these studies fail to be published or have inadequate findings as they succumb to common methodological flaws. Many of these flaws can be attributed to not having a clear definition of, or simply misunderstanding or misusing the terms stress and burnout. Other studies fail to follow the scientific method and do not consider the true intent of a stress or burnout project at the outset and therefore are not sufficiently explicit in drafting their research question. Lastly, some researchers attempt to write and publish a manuscript simply because data is available as their institution is collecting or has collected data on stress or burnout which is also ill advised. For example, the Accreditation Council for Pharmacy Education Standards (ACPE) standard 24 encourages colleges and schools to assess and correct underlying causes of ineffective learning experiences, such as "perceived stress in faculty, staff, and students." 11 Although conducting projects for accreditation purposes is legitimate and certainly benefits the institution, 
simply conducting a descriptive analysis of these data or relating it to other factors via a cross-sectional design does not constitute meaningful research in this area. Given its lack of rigor and theoretical grounding, such research would be of limited value to the Academy and would likely not get published.

The intent of this integrative review is to provide faculty members and beginning researchers interested in burnout and stress research a resource highlighting psychological theory, concise definitions of burnout, stress, and related pertinent terminology, a summary of reliable, validated, rigorous quantitative measurement tools, and suggestions for future research. Specifically, this article is focused on an individual perspective and does not address organizational measurement instruments or programs, nor qualitative methods of measurement for burnout and stress as these are beyond the scope of this review. The authors hope that this paper will help improve the rigor of burnout and stress research in pharmacy education and expand scientific inquiry and application of findings, ultimately helping to not only assess but also correct underlying causes of burnout and stress for students, faculty, and staff.

\section{METHODS}

In conducting this method review authors utilized a university librarian to assist in identifying previously published exemplar articles and validated instruments to measure burnout and stress. Database searches of PubMed, PsycInfo, CINAHL, and Web of Science were conducted. The stress MeSH terms utilized were "stress, psychological", "occupational stress", "compassion fatigue," "mental fatigue", "caregiver burden" and "stress disorders, post traumatic." The MeSH terms used to identify articles related to burnout were "burnout, psychological", "burnout, professional". To limit the results to validated instruments, the MeSH terms utilized were "reproducibility of results", "data accuracy", "dimensional measurement accuracy", and "scientific experimental error." This search strategy yielded 491 manuscripts that 2 authors independently reviewed for inclusion of measurement instruments in this review. All debatable measurement instruments for inclusion were discussed among authors utilizing a consensus process to arrive at a shared final decision.

\section{Defining Concepts Related to Occupational Stress}

Most theories of occupational stress share a few common features. ${ }^{12}$ (Figure 1) Stress is best depicted as a process rather than an emotion or event because people tend to think of it in terms of both the causes, hereafter referred to as stressors, as well as their response. ${ }^{13}$ Stressors commonly arise from the physical or psychological demands of work and include factors such as workload, time pressure, role ambiguity or conflict, organizational politics, and information and communication technologies. For students, these may manifest as course and experiential workload, exam deadlines, assignment ambiguity or misinformation, use of multiple educational technologies, and outside employment. Faculty must also manage challenges related to workload and deadlines as well as ambiguity or conflict across multiple roles (eg, school versus practice site responsibilities), academic bureaucracy, and a growing number of technology platforms (eg, email, videoconferencing, file-sharing services, collaborative workspaces).

If the response to a stressor is negative, it is referred to as a strain, and the relationship between stressors and strains is complex. ${ }^{13}$ For example, having too few stressors has been found to lead to boredom and can thwart the sense of accomplishment that results from overcoming challenges. ${ }^{14}$ Stressor response is also highly variable as some people may experience significant strain with mounting demands whereas others may thrive in such environments. Many theories of occupational stress account for this variation by recognizing an intermediate step, sometimes referred to as appraisal because it represents an individual's viewpoint or "appraisal" of a stressor as either a challenge or a hindrance. ${ }^{10}$ Challenges are viewed as being surmountable with effort (eg, workload) and can improve well-being until they exceed one's capacities. ${ }^{16-17}$ Hindrances, by contrast, are perceived as being outside a person's control (eg, organizational politics) and appear to exert only detrimental effects unless counteracted by sufficient resources (eg, supervisor support, autonomy in making decisions about one's work). ${ }^{15,17}$

Appraisal is then followed by an initial response (eg, changes in heart rate, feelings of distress) and maladaptive responses eventually result in strains, especially following prolonged exposure. Feelings of psychological distress in the short-term are often referred to informally as "stress", but they are more precisely viewed as a form of psychological strain and can lead to burnout if left unaddressed. ${ }^{13}$ Although the focus of this review is psychological strain, responses to stressors can also be physical (eg, headaches, gastrointestinal disorders) and behavioral (eg, substance use, disordered eating) and often these phenomena coexist. ${ }^{18-19}$ Both the initial response to a stressor and the eventual strain can ultimately affect organizational outcomes (eg, performance, turnover), illustrating the importance of well-being as an organizational priority even if strains are limited to a few students or employees.

Numerous personal and environmental factors can influence how a person appraises and responds to a stressor. Personal factors include personality and other dispositional traits (eg, neuroticism, locus of control) as well as skills and experiences accumulated over time, such as resilience, or one's capacity to bounce back from adversity. ${ }^{20}$ Environmental 
factors include job resources such as supervisor or coworker support, levels of autonomy or control (ie, decision latitude) in the job, and organizational culture, which can manifest as formal policies as well as unwritten norms (eg, expectations to respond to email after hours). Both personal and environmental factors can serve as the targets of interventions to improve student and employee well-being, although individual-focused approaches tend to predominate the literature. ${ }^{21}$ Given that most stressors originate from work itself, organizational interventions aimed at reducing work-related demands (eg, providing greater role clarity, streamlining technology use) or increasing work-related resources (eg, increased organizational support, greater decision latitude) are likely to be more successful in the long-term. ${ }^{18}$

\section{Burnout}

Burnout is a psychological strain resulting from exposure to chronic, unmitigated stressors. ${ }^{22}$ Although most experts agree that burnout is limited to the work environment, others have challenged this assertion based on overlap between burnout symptoms and those of depression and other mental health disorders. ${ }^{23-24}$ Despite these relationships, one of the strongest arguments in favor of conceptualizing burnout as a workplace concern is that this allows one to attribute the problem to work-related factors rather than personal failings.

Burnout is commonly conceptualized as a syndrome consisting of three dimensions: exhaustion, depersonalization and inefficacy. Exhaustion and depersonalization are thought to represent the core of burnout. ${ }^{25}$ Burnout-related exhaustion is traditionally measured in emotional terms, although some scales also include physical and cognitive fatigue. Exhaustion receives the most attention in the literature because it is the dimension that occurs most frequently; however, experts suggest that exhaustion alone is insufficient for defining burnout. ${ }^{22}$ Depersonalization (also referred to as disengagement or cynicism) refers to putting emotional distance between oneself and work, often as a response to exhaustion. ${ }^{22}$ The third dimension of burnout, inefficacy (or lack of professional accomplishment), is the least studied and some research suggests it may occur independently from exhaustion or depersonalization. ${ }^{26}$

Although burnout has been a topic of focus in pharmacy, less attention has been paid to the optimal psychological state(s) that organizations should strive to achieve, as less burnout does not necessarily mean that people are thriving at work. In pharmacy education, most have advocated for building resilience and fostering wellbeing. The term "resilience" is often used informally, but a review of stress frameworks (Figure 1) illustrates that this framing is imprecise. Resilience may offer some protection from burnout, but it is only one of numerous factors affecting the stressor response, and its association with immutable characteristics (eg, age, gender, race) suggests that it is not entirely under a person's control. ${ }^{27}$ "Well-being" is a better term, but because it spans physical, mental, and social health across all domains of life, interventions to improve overall well-being may be too broad to address burnout specifically.

Work engagement is a phenomenon that likely represents the optimal state of people at work, although experts disagree as to whether it is the opposite of burnout or simply a positive work-related state of mind. ${ }^{25}$ Work engagement is also a three-dimensional phenomenon and consists of vigor (ie, high levels of energy and persistence), dedication (ie, feeling a sense of pride and enthusiasm), and absorption (ie, being fully engrossed in one's work, sometimes referred to as "flow"). ${ }^{28}$ To ensure that the full breadth of work experiences is captured in an organization, experts recommend measuring both constructs rather than viewing low levels of burnout as representing high levels of work engagement. ${ }^{25}$

\section{Burnout Measurement Instruments}

Researchers have used a variety of validated and non-validated questionnaires to measure burnout. The Maslach Burnout Inventory (MBI), ${ }^{29}$ Oldenburg Burnout Inventory (OLBI), ${ }^{25}$ and Copenhagen Burnout Inventory $(\mathrm{CBI})^{30}$ are the most common validated instruments reported in the health professions literature.

The MBI is the most widely recognized burnout instrument. The original MBI for human services workers (MBIHSS) measured burnout in human services professionals (eg, police, clergy, social workers) and addressed three scales: emotional exhaustion, depersonalization, and personal accomplishment. The MBI has since been adapted for use with medical personnel (MBI-HSS (MP)), educators (MBI-ES), students (MBI-GS (S)), and general use (MBI-GS) by slight wording alterations of select questions and scale changes to reflect the respective environments and group being studied. For example, the MBI-GS (S) scales are exhaustion, cynicism (toward studies), and professional efficacy (in relation to school accomplishments). The MBI instruments contain either 16 or 22 items and take 10-15 minutes to complete. The MBI is proprietary and thus involves a fee for use: (https://www.mindgarden.com/117-maslach-burnout-inventory-mbi)

The OLBI was developed to overcome some psychometric issues of the MBI and measures two dimensions of burnout: exhaustion and disengagement from work. The OLBI exhaustion dimension differs from the MBI in that it includes physical and cognitive aspects of exhaustion, not just affective. ${ }^{31}$ In the OLBI, disengagement includes the depersonalization component, but also addresses a general distancing of oneself from work and work activities and not just the people with whom one works. ${ }^{25}$ Also, unlike the MBI, the OLBI does not include personal accomplishment for 
reasons described in detail previously. ${ }^{32}$ The OLBI instrument contains 16 items and is available as an appendix in a study by Demerouti, et al. ${ }^{25}$

The CBI was also developed as an alternative to the MBI and consists of three sub-dimensions: personal burnout, work-related burnout, and client-related burnout. ${ }^{30}$ The philosophical core of the CBI is the attribution of fatigue and exhaustion to specific aspects of a person's life. Compared to MBI and OLBI, one major difference of the CBI is that it does not measure depersonalization/cynicism because the authors view them as a separate phenomenon. ${ }^{30}$ The CBI is a 16-item instrument and available free online: (https://nfa.dk/da/Vaerktoejer/Sporgeskemaer/Sporgeskema-til-maaling-afudbraendthed/Copenhagen-Burnout-Inventory-CBI)

The Burnout Assessment Tool (BAT) is the most recent instrument developed as an alternative to the MBI. ${ }^{33}$ The BAT is based on a reconceptualized definition of burnout that includes four primary dimensions: exhaustion, mental distance, emotional impairment and cognitive impairment and three secondary dimensions: depressed mood, psychological distress, and psychosomatic complaints. The BAT consists of 33 items and measures both the core and secondary dimensions, producing a single burnout score as opposed to the multiple scale scores of the MBI and OLBI. The questionnaire is available as an appendix in the original validation manuscript. ${ }^{33}$

In addition to the three primary instruments, a subscale of the Professional Quality of Life Scale (ProQol) ${ }^{34}$ has also been used to measure burnout in several studies. ${ }^{35}$ The ProQol is a 30-item instrument with ten questions each addressing compassion satisfaction, secondary traumatic stress, and burnout. However, the burnout subscale has been criticized for lacking adequate construct validity and therefore should be used with caution. ${ }^{36-37}$

Other researchers have attempted to measure burnout with a single question: "Overall, based on your definition of burnout, how would you rate your level of burnout?" 38 Because of the simple administration and no-cost availability it was offered as an alternative to the MBI. While a single-item measure may approximate burnout fairly well, one criticism is that it predicts high emotional exhaustion, but not low exhaustion or depersonalization. ${ }^{39}$

Researchers certainly may find other measures of burnout in the literature. A 2018 systematic review by O'Connor found eight validated measures of burnout cited in the literature between 1997 and $2017 . .^{40}$ These measures including number of times cited included the previously mentioned MBI $(n=54), \operatorname{CBI}(n=3)$ and OLBI(n=2) instruments, in addition to the Pines Burnout Measure $(n=3)$, the Psychologists Burnout Inventory $(n=2)$, the Organisational Social Context Scale (OSCS) $(\mathrm{n}=1)$, the Professional Quality of Life Scale (ProQOL III)( $\mathrm{n}=1)$ and the Children's Services Survey- emotional exhaustion subscale $(n=1)$. Ultimately, researchers should seek to use a tool most suitable to their specific research question.

\section{Measuring Stress}

As described above, "stress" is often used to describe the short-term feelings of psychological strain that can result from situations in which a person perceives their current resources (physical and psychological) are insufficient to meet the demands. ${ }^{41}$ Similar to burnout, stress has been studied broadly in health care, K-12 and college students, and pharmacy education. Common struggles for measuring stress include those mentioned previously including the complex and dynamic relationship of stressors, strain, stress responses, and individualized differences in responses to name a few. "Stress" even among researchers in the field is now seen as too nebulous of a term to be used when referring to such a variety of things. ${ }^{42}$ To combat this, researchers have defined a stress typology including considerations for timing, life stage, stressor attributes and responses, and the timeframe for measurement to assist researchers. ${ }^{43}$ Additionally, researchers seeking to evaluate stress are encouraged to review items from the MacArthur Foundation Research Network on SES \& Health and the National Institute on Aging supported stress measurement network which includes a toolbox of 30 psychological measurement areas ranging from caregiver stress and financial strain to salivary cortisol with included notes and/or scales for measurement: (https://macses.ucsf.edu/whatsnew/default.php)

(https://www.stressmeasurement.org/)

\section{Stress Measurement Instruments}

One of the most commonly employed scales to assess a respondents subjective perception of stress is the Perceived Stress Scale (PSS) which is a validated scale available in a 4, 10, and 14 question version and has participants answer questions regarding their feelings and thoughts during the past month on a 4-point scale of never to very often with higher scores correlating to greater stress. ${ }^{44}$ Refer to Table 2 for the 14 item PSS.

The Stress Overload Scale is another commonly used validated tool and consists of a 30-item scale used to evaluate overload when an individual experiences demands that exceed available resources. Respondents answer questions evaluating their stress in the past week using a 5-point scale. ${ }^{45}$ A recently developed 10 -item version (Table 3 ) may also be used to evaluate acute stress overload, ie, when demands are greater than resources. ${ }^{46}$ 
Another tool to evaluate global stress is the Stress In Context questionnaire. This validated questionnaire specifically attempts to contextualize stress perception based on the environment, ie, work, home, etc. This questionnaire is relatively new, but has demonstrated good correlations to the PSS in evaluating self reported measures of stress, wellbeing, and health. ${ }^{47}$

Other measures of "stress" exist, but are beyond the scope of this review. Given the complexity of this topic, we encourage researchers to use the term stress to refer to the dynamic stimulus-response process depicted in Figure 1 and use terms such as stressors and strains (or responses, if studying more than just maladaptive reactions) to refer to the various components of this relationship. Researchers should also specify their stressors and strains of interest, as a variety of validated instruments are often freely available for measuring these constructs. Another important consideration is to determine whether global or facet-level measures of strain are more appropriate. For example, a global measure such as the PSS (or OLBI if interested in longer-term effects) might be appropriate for assessing the effects of implementing a new exam scheme (ie, workload, time pressure) on student well-being whereas a study assessing the effects of student loans (ie, a financial stressor) might include both a global measure as well as a measurement specific to financial strain, which could be as simple as a single item. ${ }^{48}$

\section{DISCUSSION}

Burnout and stress are two popular areas of research inquiry in pharmacy education that when viewed in context are pieces of a larger, more complex and interconnected conceptualization of stress and well-being. Research into these areas is critical to the Academy as the profession of pharmacy, students, faculty, and staff all are impacted by varying levels of strain from multiple sources. As discussed in this integrative review, researchers must evaluate more than simply stress or burnout in order to produce scholarship that is both publishable and will help advance this research area. An example that researchers could use as guidance is a 2018 study conducted by Gordon, et al. in which the investigators explored the effects of a job crafting intervention among health care professionals. ${ }^{49}$ The research was grounded in established theory (eg, the job demands-resources model) and the investigators explicitly defined and measured their outcomes of interest (eg, the exhaustion domain of burnout) using validated instruments (eg, Oldenburg Burnout Inventory). Investigators introduced an evidence-based intervention (ie, training health care professionals how to craft their jobs) and then measured the effects of the intervention on participants over time (ie, a longitudinal analysis). Notably, the study did not involve pharmacists, providing an opportunity for future research given that pharmacists might craft their jobs differently than physicians or nurses.

In this article, we have provided an overview of stress and burnout and referenced literature where additional information about the frameworks describing these phenomena can be found. We have also provided a list of validated instruments commonly used to evaluate stress and burnout (Table 1). We hope that the information contained in this review will assist researchers in improving the rigor of their research. As illustrated in the example above,critical elements that researchers should follow include following the scientific method, asking specific research questions that are rooted in theory, correctly defining and measuring the outcome of interest with validated/reliable instruments, and taking into consideration the dynamic relationship between stressors, strain, and individual factors in relation to timing of data collection, etc. Lastly, authors hope that this review will not only encourage further exploration into measurement of burnout and stress, but expand this research beyond simple measurement to also include novel ways to address these issues. It is our hope that this research will extend well beyond individualized traits and stress response (ie, resilience) and will include evaluation of organizational practices impacts on burnout and stress including the reporting of theory guided organizational changes impacts on burnout and stress.

\section{CONCLUSION}

Burnout and stress are important research topics that warrant considerable attention within the academy. Adherence to the research method and usage of validated reliable surveys will improve the rigor and utility of research findings in this area. Further research into burnout and stress remedies including organizational practices is needed.

\section{REFERENCES}

1. Bria M, Baban A, Dumitrascu DL. Systematic review of burnout risk factors among European healthcare professionals. Cogn Brain Behav. 2012;16(3):423-452.

2. Marshall LL, Allison A, Nykamp D, et al. Perceived stress and quality of life among doctor of pharmacy students. Am J Pharm Educ. 2008;72(6):137. doi:10.5688/aj7206137. 
3. Gupchup GV, Borrego ME, Konduri N. The impact of student life stress on health-related quality of life among doctor of pharmacy students. Coll Stud J. 2004;38(2):292-301.

1. 4.Votta RJ, Benau EM. Predictors of stress in doctor of pharmacy students: results from a nationwide survey. Curr Pharm Teach Learn. 2013;5(5):365-372. doi:10.1016/j.cpt1.2013.06.014.

4. Saul B, Andrews S, Hartman J, et al. Assessment of stress and quality of life in non-native and native Englishspeaking pharmacy students. Am J Pharm Educ. 2021;85(6):410-414. doi: 10.5688/ajpe8351.

5. Kennedy DR, Clapp P, DeLuca JL, Filtz T, Kroon L, et al. Enhancing pharmacy faculty well-being and productivity while reducing burnout. Am J Pharm Educ. 2021, 8764; doi: 10.5688/ajpe8764.

6. Sabagh Z, Hall NC, Saroyan A. Antecedents, correlates and consequences of faculty burnout. Educ Research. 2018; 60(2): 131-156.

7. Darbishire P, Isaacs AN, Miller ML. Faculty burnout in pharmacy education. Am J Pharm Educ. 2020; 84(7). Doi: 10.5688/ajpe7925.

8. Wallace JE, Lemaire JB, Ghali WA. Physician wellness: a missing quality indicator. Lancet 2009 Nov 14;374(9702):1714-21. doi:10.1016/S0140-6736(09)61424-0

9. Pangioti M, Panagopoulou E, Bower P. Controlled interventions to reduce burnout in physicians: a systematic review and meta-analysis. JAMA Intern Med. 2017;177(2):195-205. doi:10.1001/jamainternmed.2016.7674

10. Accreditation Council for Pharmacy Education. Guidance for the accreditation standards and key elements for the professional program in pharmacy leading to the doctor of pharmacy degree. Available at: https://www.acpeaccredit.org/pdf/GuidanceforStandards2016FINAL.pdf. Accessed June 10, 2021.

11. Beehr T. Chapter 1: An organizational meta-model of occupational stress. In: Cooper C, ed. Theories of organizational stress. Oxford;1998:6-26

12. Jex SM, Beehr TA, Roberts CK. The meaning of occupational stress items to survey respondents. J Appl Psychol. 1992;77(5):623-628.

13. Lock EA, Latham GP. Building a practically useful theory of goal setting and task motivation. Am Psychol. 2002;57(8):705-717.

14. Dawson KM, Obrien KE, Beehr TA. The role of hindrance stressors in the job demand-control-support model of occupational stress: a proposed theory revision. J Organiz Behav. 2016;37:397-415.

15. Toker S, Shirom A, Melamed S, Armon G. Work characteristics as predictors of diabetes incidence among apparently healthy employees. J Occup Health Psychol. 2012;17(3):259-267.

16. Schilbach M, Baethge A, Rigotti T. Do challenge and hindrance job demands prepare employees to demonstrate resilience? J Occup Health Psychol. 2021;26(3):155-174.

17. Glazer S, Liu C. Work, stress, coping, and stress management. In: Oxford research Encyclopedia, psychology.Oxford; 2011;1-43. doi: 10.1093/acrefore/9780190236557.013.30.

18. Spector PE, Chen PY, OConnell BJ. A longitudinal study of relations between job stressors and job strains while controlling for prior negative affectivity and strains. J Appl Psychol. 2000;85(2):211-218.

19. Windle G, Bennett KM, Noyes J. A methodological review of resilience measurement scales. Health Qual Life Outcomes. 2011;9(8):1-18.

20. Richardson KM. Managing employee stress and wellness in the new millennium. J Occup Health Psychol. 2017;22(3):423-428.

21. Maslach C, Schaufeli WB, Leiter MP. Job burnout. Annu Rev Psychol. 2001;52:397-422.

22. Bianchi R, Schonfeld IS, Laurent E. Burnout: moving beyond the status quo. Int J Stress Manag. 2019;26(1):3645.

23. Meier ST, Kim S. Meta-regression analyses of relationships between burnout and depressions with sampling and measurement methodological moderators. J Occup Health Psychol. 2021. doi: 10.1037/ocp0000273

24. Demerouti E, Mostert K, Bakker AB. Burnout and work engagement: a thorough investigation of the independency of both constructs. J Occup Health Psychol. 2010;15(3):209-222.

25. Lee RT, Ashforth BE. A meta-analytic examination of the correlates of the three dimensions of job burnout. $J$ Appl Psychol. 1996;81(2):123-133.

26. Davidson JRT. Connor-Davidson resilience scale (CD-RISC) manual. Unpublished. 2020, accessible at www.cd.risc.com

27. Schaufeli WB, Salanova M, Gonzalez-Roma V, Bakker AB. The measurement of engagement and burnout: a two sample confirmatory factor analytic approach. J Happiness Stud. 2002;3:71-92.

28. Maslach C, Jackson SE, Leiter MP. Maslach Burnout Inventory, Manual (3rd ed.). 1996;Palo Alto, CA: Consulting Psychologists Press. 
29. Kristensen TS, Borritz M, Villadsen E, Christensen KB. The Copenhagen Burnout Inventory: A new tool for the assessment of burnout. Work Stress. 2005;19:192-207.

30. Demerouti E, Bakker AB, Vardakou I, Kantas A. The convergent validity of two burnout instruments: Amultitrait-multimethod analysis. Eur J Psychol Assess. 2003;19(1):12-23.

31. Bakker AB, Demerouti E, Verbeke W. Using the job demands-resources model to predict burnout and

2. performance. Human Resource Management. 2004;43(1):83-104.

32. Schaufeli WB, Desart S, De Witte H. Burnout Assessment Tool (BAT)—Development, Validity, and Reliability. Int J Environ Res Public Health. 2020;17(24):9495. https://doi.org/10.3390/ijerph17249495

33. Stamm, B. H. (2005). The ProQol manual. Brooklandville, MD: Sidran Press.

34. Doolittle BR. Association of burnout with emotional coping strategies, friendship, and institutional support among internal medicine physicians. J Clin Psychol Med Settings. 2020;28: 361-367.

35. Heritage B, Rees CS, Hegney DG. The ProQOL-21: A revised version of the Professional Quality of Life (ProQOL) scale based on Rasch analysis. PloS one. 2018 Feb 28;13(2):e0193478.

36. Hemsworth D, Baregheh A, Aoun S, Kazanjian A. A critical enquiry into the psychometric properties of the professional quality of life scale (ProQol-5) instrument. Appl Nurs Res. 2018;1(39): 81-88.

37. Dolan ED, Mohr D, Lempa M, et al. Using a single item to measure burnout in primary care staff: a

3. psychometric evaluation. J Gen Intern Med. 2015;30:582-587

38. Waddimba AC, Scribani M, Nieves MA, Krupa N, May JJ. Validation of single-item screening measures for provider burnout in a rural health care network. Eval Health Prof. 2015;39:215-25.

39. O'Connor K, Neff DM, Pitman S. Burnout in mental health professionals: A systematic review and meta-analysis of prevalence and determinants. European Psychiatry. 2018;53:74-99.

40. Cohen S, Gianaros P, Manuck S. A stage model of stress and disease. Perspect Psychol Sci. 2016;11(4):456-463.

41. Crosswell AD, Lockwood KG. Best practices for stress measurement: how to measure psychological stress in health research. Health Psychol Open. 2020;7(2). https://dx.doi.org/10.1177\%2F2055102920933072

42. Epel ES, Crosswell, AD, Mayer SE, Prathr AA, Slavich GM, Puterman E, Mendes WB. More than a feeling: a unified view of stress measurement for population science. Front Neuroendocrinol. 2018;49:146-169.

43. Cohen S, Kamarck T, Mermelstein R. A global measure of perceived stress. J Health Soc Behav. 1983 Dec;24(4):385-396.

44. Amirkhan JH. Stress overload: a new approach to the assessment of stress. Am J Community Psychol. 2012 Mar;49(1-2):55-71.

45. Amirkhan JH. A brief stress diagnostic tool: the short Stress Overload Scale. Assessment. 2018 Dec;25(8):10011013.

46. Mayer SE, Epel ES, Slavich GM Mendes WB. Psychometric validation of the Stress in Context questionnaire: links with psychological and physiological measures of stress and health. https://164f89be-6459-4649-bea5b3d3fcc248ee.filesusr.com/ugd/890fda_c67115db51a84b399b9cf189dd1d649e.pdf. Accessed October 1, 2021.

47. Okechukwu CA, El Ayadi AM, Tamers SL, Sabbath EL, Berkman L. Household food insufficiency, financial strain, work-family spillover, and depressive symptoms in the working class: the work, family, and health network study. Am J Public Health. 2012 Jan;102(1):126-133.

48. Gordon HJ, Demerouti E, LeBlanc PM, Bakker AB, Bipp T, Verhagen A. Individual job redesign: job creating interventions in healthcare. J Voc Behavior 2018;104:98-114. 
Table 1. Comparison of Validated Instruments Measuring Burnout and Stress

\begin{tabular}{llll} 
Instrument & $\begin{array}{l}\text { Construct } \\
\text { Measured }\end{array}$ & Technical Considerations & General Comments \\
\hline
\end{tabular}

\section{Maslach Burnout Inventory -}

Educators Survey (MBI-ES)

Maslach Burnout Inventory - General Burnout
Survey for
Students (MBI-GS(S))

Maslach Burnout Inventory - Human Burnout

Services Survey for Medical

Personnel (MBI-HSS(MP))

\section{Oldenburg Burnout Inventory (OLBI) Burnout}

Copenhagen Burnout Inventory (CBI)

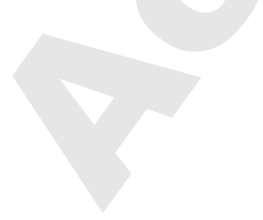

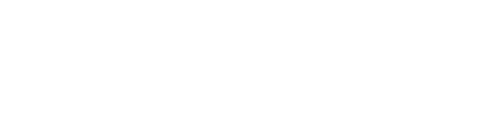

Burnout Assessment Tool (BAT)

Burnout
Burnout

Validated by extensive
research

High internal reliability

Fee-based

Burnout

Validated by extensive research

High internal reliability

Fee-based

Validated by extensive research

High internal reliability

Fee-based

Includes both positive and negative framed items

Available in appendix of original article for free

Limited benchmark data for United States

High internal reliability

Validated

High internal reliability

Available online for free

Cutoff scores not based on evidence

Limited benchmark data for United States Convergent validity with
both MBI and OLBI
Adaptation of the original MBI-HSS to be used with educators, administrators, and other education personnel

Measures Emotional Exhaustion, Depersonalization, and Personal

Accomplishment

Adaptation of the MBIGS to be used with college students

Measures Exhaustion, Cynicism, and Professional Efficacy

Adaptation of the original MBI-HSS to be used with medical personnel

Measures Emotional Exhaustion,

Depersonalization, and Personal

Accomplishment

Based on Job demands Job resources model

Measures Exhaustion and Disengagement

Measures Physical and Psychological fatigue, Physical and Psychological Fatigue related to work, and Client-related Burnout

Based on a comprehensive conceptualization of burnout derived from 
original article for free

More testing is needed to fully validate the instrument

Stress

Perceived Stress Scale (PSS)

Broad relative measure
Free

Broadly used in stress literature

Gold standard to which other measures use for validity

Measures stress over last month

Strictly evaluates stress

No formally researched rubric for stratification of respondents

Stress

Excellent internal validity

Free

Excellent internal consistency

Extensive psychometric validity and reliability

Can stratify respondents by risk categories

Appropriate for community research use

Less vulnerable to social desirability and negative affectivity biases

30 item version may be cumbersome but less susceptible to self-report biases and weaknesses

Criterion validity comparable to full SOS interviews with practitioners

Measures Exhaustion, Mental Distancing, Cognitive Impairment, Emotional Impairment, Depressed Mood, Psychological Distress, and Psychosomatic Complaints

Literature links to selfreported health, biological markers of stress/disease, and psychological stress
The first entirely empirically developed survey to evaluate stress

Has proven cross validity with several common stress instruments including PSS

Allows for both categorical and continuous evaluation of data (categories of personal vulnerability and event load can be used to create a four category diagnostic matrix) 
Concurrent and predictive validity for identifying sicker respondents and predicting future signs of illness

Vulnerable to social desirability and negative affectivity biases

Free

Stress in Context (SIC) Questionnaire Stress

Effort-Reward Imbalance (ERI)Questionnaire
Assesses stress perceptions in specific contexts

Potentially more useful in low income populations or others experience chronic adversity

Equivalent to the PSS in self-report measures of psychological distress, well-being, and selfreported health

Free with permission from researcher

Work Stress

2 versions (long and short) with 22 and 16 items respectively

Convergent and criterion validity, factorial structure, reliability and sensitivity to change over time

Used in a number of largescale studies

Free

Susceptible to Self reported measure biases, etc.
New measure that hasn't been thoroughly evaluated
Not intended to be a clinical screening or outcome measure 
Table 2. Perceived Stress Scale- 14 item (PSS-14) $)^{39}$

The questions in this scale ask you about your feelings and thoughts during THE LAST MONTH. In each case, please indicate your response by circling HOW OFTEN you felt or thought a certain way.

1. In the last month, how often have you been upset $\quad 0=$ never; $1=$ almost never; $2=$ sometimes; $3=$ fairly often; because of something that happened unexpectedly? 2. In the last month, how often have you felt that you were unable to control important things in your life? 3. In the last month, how often have you felt nervous and "stressed"?

4. In the last month, how often have you dealt successfully with irritating life hassles?

5. In the last month, how often have you felt that you were effectively coping with important changes that were occurring in your life?

6. In the last month, how often have you felt confident about your ability to handle your personal problems?

7. In the last month, how often have you felt that things were going your way?

8. In the last month, how often have you found that you could not cope with all the things that you had to do? 9. In the last month, how often have you been able to control irritations in your life?

$4=$ very often

$0=$ never; $1=$ =almost never; $2=$ =sometimes; 3 =fairly often; $4=$ very often

$0=$ never; $1=$ almost never; $2=$ sometimes; $3=$ fairly often; $4=$ very often

$0=$ never; $1=$ =almost never; $2=$ sometimes; 3 =fairly often; $4=$ very often

$0=$ never; $1=$ =almost never; $2=$ sometimes; $3=$ fairly often; $4=$ very often

$0=$ never; $1=$ =almost never; $2=$ sometimes; $3=$ fairly often; $4=$ very often

$0=$ never; $1=$ =almost never; $2=$ =sometimes; $3=$ fairly often; $4=$ very often

$0=$ never; $1=$ =almost never; $2=$ =sometimes; 3 =fairly often; $4=$ very often

$0=$ never; $1=$ =almost never; $2=$ sometimes; $3=$ fairly often; $4=$ very often

10. In the last month, how often have you felt that you were on top of things?

11. In the last month, how often have you been angered because of things that happened that were outside of your control?

12. In the last month, how often have you found yourself thinking about things that you have to accomplish?

13. In the last month, how often have you been able to control the way you spend your time?

14. In the last month, how often have you felt difficulties were piling up so high that you could not

$0=$ never; $1=$ almost never; $2=$ sometimes; $3=$ fairly often; $4=$ very often

$0=$ never; $1=$ almost never; $2=$ sometimes; $3=$ fairly often; $4=$ very often

$0=$ never; $1=$ almost never; $2=$ sometimes; $3=$ fairly often; $4=$ very often

$0=$ never; $1=$ =almost never; $2=$ =sometimes; $3=$ fairly often; $4=$ very often

$0=$ never; $1=$ =almost never; $2=$ sometimes; $3=$ fairly often; $4=$ very often overcome them?

To Score:

1. Reverse score items 4, 5, 6, 7, 9, 10, and $13(0=4,1=3$, etc.).

2. Add together scores on all 14 items including the "reversed" scored items.

3. Final scores range from $0-56$ with higher scores indicating higher stress. 
Table 3. Stress Overload Scale- Short (SOS-S) $)^{40}$

IN THE PAST WEEK have you felt:

1. ... inadequate?

Not at all $\quad$ A lot

2. ... swamped by your responsibilities?

$\begin{array}{lllll}1 & 2 & 3 & 4 & 5\end{array}$

Not at all A lot

3. ... that the odds were against you?

$\begin{array}{lllll}1 & 2 & 3 & 4 & 5\end{array}$

Not at all A lot

$\begin{array}{lllll}1 & 2 & 3 & 4 & 5\end{array}$

4....that there wasn't enough time to get to everything? Not at all A lot

5. ... like nothing was going right?

$\begin{array}{lllll}1 & 2 & 3 & 4 & 5\end{array}$

Not at all A lot

$\begin{array}{lllll}1 & 2 & 3 & 4 & 5\end{array}$

6. ... like you were rushed?

Not at all A lot

$\begin{array}{lllll}1 & 2 & 3 & 4 & 5\end{array}$

7. ... like there was no escape?

Not at all A lot

8. ... like things kept piling up?

9. ... like just giving up?

$\begin{array}{lllll}1 & 2 & 3 & 4 & 5\end{array}$

Not at all A lot

$\begin{array}{lllll}1 & 2 & 3 & 4 & 5\end{array}$

Not at all A lot

$10 . .$. like you were carrying a heavy load? $\begin{array}{lllll}1 & 2 & 3 & 4 & 5\end{array}$

Not at all A lot

$\begin{array}{lllll}1 & 2 & 3 & 4 & 5\end{array}$ 